le pamphlet touche ici à la question épineuse de l'usage que peut faire l'humanisme de la philosophie antique. Ce pamphlet est le plus complexe et le plus long des trois pamphlets réunis dans cette édition.

Il convient de dire pour terminer que l' Advertissement et l' Histoire avaient déjà fait l'objet d'une édition accompagnée d'une étude par Francis Higman. L'édition que nous présentons ici a le mérite de réunir trois textes, dont le dernier, la Response, est ici édité pour la première fois, qui avaient été publiés de manière anonyme au $\mathrm{XVI}^{\mathrm{e}}$ siècle. Les auteurs attribuent ces trois textes à Calvin de manière tout à fait convaincante par l'usage de la philologie. Il s'agit d'une excellente édition, avec un appareil critique et bibliographique considérable. Chaque pamphlet est présenté par une introduction qui explique les causes et les circonstances de sa parution, l'originalité du texte, et le conflit idéologique qui existe entre Calvin et ses adversaires. Une grande et belle contribution à la pensée et à l'œuvre de Calvin.

CHRISTIAN FANTONI, University of South Alabama

\title{
La Méditation au XVII siècle. Rhétorique, art, spiritualité
}

Sous la direction de Christian Belin

Paris : Champion, 2006, 275 pp.

Qu'elle soit en prose ou en vers, catholique ou protestante, la méditation religieuse n’a pas été négligée par la critique. Il suffit pour s'en persuader de consulter la bibliographie de cet ouvrage. Du même coup, elle a éclipsé un peu ce qui ne relevait pas directement de la spiritualité. Christian Belin, le maitre d'œuvre de cet ouvrage, a estimé qu'on la comprendrait mieux en la confrontant avec d'autres langages. Il a surtout voulu considérer la méditation en elle-même. Voilà pourquoi il a convié à cette réflexion non seulement les littéraires et les théologiens, traditionnellement invités à des travaux de ce genre, mais aussi les philosophes, les musicologues et les historiens de l'art. On ne saurait trop le remercier pour cette ouverture, car les études qu'il a réunies forment un ensemble d'une exceptionnelle richesse.

Que le lecteur se rassure. Le genre même de la méditation n'est pas oublié grâce à Véronique Ferrer (« Les Méditations sur les Psaumes d'Agrippa d'Aubigné ») et à Véronique Adam (« La méditation poétique : un méditant-Narcisse »). Richard Parish la convoque lui aussi dans son article sur « Polémique et poétique dans la méditation catholique en prose ». D'Aubigné a toujours vanté ce qu'il appelle le « langage de Canaan », opposé à toutes les subtilités maniéristes en vogue de son temps, à leur « affectation », à leur « hypocrisie ». C'est ce langage-là que 
doivent cultiver les écrivains réformés, surtout quand il écrivent des Méditations. Celles $\mathrm{du}$ « Bouc du désert » illustrent ce modèle tout en offrant « un écho modulé des Psaumes $\gg$. Elles ne renoncent pas au commentaire (sur la prédestination, par exemple). Mais s'il oubliait son temps et les multiples reniements dont il a été le témoin scandalisé, d'Aubigné ne serait plus lui-même. Il pratique donc une « lecture contemporaine du texte sacré » (44). Avec Véronique Adam, l'objet de la recherche déjà se transforme. C'est à la méditation poétique telle que la pratiquent Tristan, Etienne Durand, Godard et quelques autres qu'elle a choisi de s'intéresser. Cette méditation-là peut être religieuse. Le plus souvent, elle est inspirée par les peines d'amour et la mélancolie. Si le méditant pratique l'introspection, il se donne surtout à voir dans sa méditation. En fermant les yeux, il lui arrive de discerner en lui « la présence de Dieu » (141). Mais il préfère laisser miroiter « l'espace méditatif $\gg$, symbolisé par la solitude et les ruisseaux dont il s'entoure. Alors que le croyant tente de s'adresser à Dieu, les poètes retenus par Véronique Adam n'entendent plus guère que leur propre voix, si bien qu'on peut parler à propos de leurs œuvres de « contre-méditations » (150). Encore trop peu connu, Abraham de Vermeil (1555-1620 ?) instaure un «étrange dialogue avec ses propres pensées » (150). La méditation engendre ainsi la mélancolie, à moins que ce ne soit l'inverse. Selon la belle formule de Véronique Adam, le méditant ne s'intéresse plus qu'à la « seule part d'infini que Dieu lui a concédée : sa pensée » (153).

Catholiques et protestants n'ont pas la même idée de la méditation. Mais il serait naï de croire que les catholiques l'envisagent tous de la même manière. C'est ce que démontre Robert Parish. Fénelon avait tendance à faire de la méditation un usage contemplatif, tandis que Bossuet lui assigne une fin plus modeste : changer le cœur du croyant, l'amener à une véritable metanoia. Elle doit « détourner du monde et $[\ldots]$ stimuler l'action caritative » (93); elle comporte une « réflexion sur la finitude de la vie ». Comment le méditant pourrait-il être indifférent à son salut, comme le voudrait l'évêque de Cambrai ? Mais si telle est bien sa finalité, la méditation appartient-elle encore à la littérature ? Richard Parish répond par l'affirmative à cette question et rapproche en passant la prose et les vers. Il met notamment en lumière la façon dont les auteurs exploitent les « jeux de voix », créant ainsi une pieuse polyphonie où l'on entend les paroles du Christ et de la Vierge, ainsi que celle, inquiète, du méditant. Ainsi conçue, la méditation devient une médiation (101). Elle dispose au salut.

C'est bien aussi de progression spirituelle qu'il s'agit dans la méditation des gens de robe, étudiée avec beaucoup de finesse par Bruno Petey-Girard. Voilà des hommes qui ont une haute idée de leur charge. C'est pourquoi ils font grand cas 
de la méditation et participent à leur manière au renouveau de la spiritualité. Le bien-dire en effet s'acquiert grâce à la méditation, compagne de l'apprentissage (49). Avant de le faire apparaitre au-dehors, il faut intérioriser le Verbe. S'agit-il de sacrifier, finalement, à l'apparence ? Pas le moins du monde. Qu' ils le sachent ou non, ces parlementaires parfois somptueux sont les héritiers de la tradition monastique. Ils cherchent à trouver leur propre voix grâce à la rumination de celle des autres. La méditation devient alors une quête intérieure. Qu'importent alors les palais du Grand Siècle !

Avec les contributions de Nathalie Grande et Georges Forestier, on déniche la méditation là où, à première vue, elle ne devait pas se trouver. D’abord dans le roman classique. Il fallait de l'audace pour présenter le roman comme un « genre spirituel ». Pourtant, Nathalie Grande nous convainc. Romanciers et spirituels, au XVII ${ }^{\text {ème }}$ siècle, s'opposent beaucoup moins qu'on ne le croit. Les exemples de JeanPierre Camus et de Fénelon montrent qu'on peut être l'un et l'autre. Les spirituels, en racontant leur vie, ont parfois adopté une écriture romanesque (sainte Thérèse). Le plus neuf, peut-être, dans ces pages, se trouve dans l'étude des « pauses méditatives $\gg$, nombreuses dans le roman de l'époque. Sous l'influence de la littérature pastorale, elles prennent parfois le nom de « rêverie ». Nathalie Grande donne l'exemple remarquable d'un passage de la Clélie, où Madeleine de Scudéry définit ce qu'elle appelle la « rêverie douce », celle où on laisse « errer son esprit ». Cette faculté n'est pas donnée à tout le monde, mais il arrive à certains personnages de connaître cet état de grâce : « D’abord, tout le monde parla assez haut, puis peu à peu chacun se taisant, ou parlant bas, on n'entendait plus que le battement des rames, qui, retombant dans l'eau dans un mouvement mesuré, faisait un certain bruit propre à exciter la rêverie $\gg$ (188). On croirait lire le Rousseau de la Cinquième Promenade. Ce genre de rêverie n'appartient à la méditation que si on donne à celle-ci un sens assez large. Mais l'idée est très convaincante. Nathalie Grande l'aurait été encore davantage si elle avait tenu compte de Don Quichotte, où abondent ces pauses méditatives. Remercions-la aussi pour le beau paragraphe consacré à La Princesse de Clèves, où, à la suite de Philippe Sellier, elle voit une sorte de « saisissante vanité littéraire $\gg(190)$.

La méditation, on la trouve aussi dans le théâtre du Grand Siècle dont s'occupe ici Georges Forestier. Grâce peut-être aux sentences dont la tragédie est émaillée, mais qui ne sont pas du goût de tout le monde. C'est en fait l'action dramatique elle-même, qui invite à la méditation, sans laquelle la catharsis n'opère pas. Elle trouve un espace d'élection dans les stances (170), qui prennent la relève du chœur de la tragédie humaniste. Sous nos yeux, « le personnage se livre $[\ldots$ a à une forme 
(profane évidemment) d'exercice spirituel » (175) que l'on peut rapprocher, sans arbitraire, de la définition ignatienne.

Prose et poésie ; roman et théâtre : la méditation envahit tout. Mais nous ne sommes pas au bout de nos surprises. Voici que la peinture et la musique entrent en scène. On se doutait, depuis le livre de Marc Fumaroli ${ }^{1}$, que la peinture du XVII ${ }^{\text {ème }}$ siècle possédait quelque chose de méditatif. Anne le Pas de Sécheval démontre d'abord que l'image peinte joue un rôle dans la méditation chrétienne, mais sa contribution va beaucoup plus loin. Quels sont les tableaux, se demande-t-elle, qui peuvent orienter le regard vers les réalités intérieures (202) ? Parmi d'autres, ceux de Philippe de Champaigne. D’une manière plus générale, «il est [ ... ] légitime de parler de méditation quand le tableau dilate le sujet de la représentation en suggérant derrière le sens littéral un réseau d'allusions narratives et de significations théologiques » (201). Mais il faut mettre le spectateur sur la voie : les peintres de l'« école du silence » semblent se souvenir du conseil d'Alberti : placer au premier plan un personnage qui nous regarde et attire notre attention sur la scène représentée, comme dans cette «Déploration du Christ », de Reynaud Levieux, objet d'une analyse convaincante. On pouvait aussi songer aux belles pages d'E. Panofsky ${ }^{2}$ sur la peinture de dévotion, qui commence au XV ${ }^{\text {ème }}$ siècle ; peut-être même au genre pictural très particulier que constituent les « Conversations sacrées », à ces personnages hors du temps et de l'histoire qui méditent quelque part sur l'histoire du salut.

Que la musique, elle aussi, puisse être méditative, on l'admet bien volontiers : il suffit de penser, par exemple, à Jean-Sébastien Bach. Il n'est pas mauvais, cependant, de préciser les choses, ce que font Maya Suemi Lemos et Anne Piéjus. La première analyse de très près un motet qui a pour titre «Contemptus mundi » et pour auteur Giacomo Carissimi (1605-1674), maître de chapelle au Collegio germanico de la compagnie de Jésus et à San Apollinaire, à Rome. On y entend la voix de Salomon revenu de tout, et le fameux vanitas vanitatum. La musique a pour but de plonger l'auditoire dans la mélancolie et la consternation (252). Atteint-elle vraiment son but ? On peut se le demander. Dieu, finalement, est absent de cette œuvre, envoûtée par la vanité. « Censée nous conduire au divin par le dévoilement du visible, tout ce qu'elle peut nous montrer pourtant est le visible lui-même » (254). « Nous restons toujours dans le temps, emprisonnés dans la sphère humaine des passions coupables » (255). Anne Piéjus, de son côté, s'intéresse à l'oratorio de l'époque, sous ses deux espèces : historiae et dialogues, qui doivent ébranler les passions et faire jaillir les larmes du repentir. L'oratorio respecte sans doute le texte sacré, ce qui n'empêche pas la sensualité des ornements. Souvent, on est loin de la liturgie. La musique aura atteint son but si le fidèle opère 
un retour sur lui-même. Parfois, elle vise plus haut et la méditation se transforme alors en contemplation, par exemple dans les vocalises sur le $\ll \mathrm{o} \gg$ des antiennes de Noël. Alors, la musique se libère de la parole (232), elle accompagne et précède le silence qui la suit.

Toutes ces analyses qui se suffisent à elles-mêmes sont éclairées par trois contributions plus théologiques ou philosophiques. Celle de Ralph Deconinck a pour sujet le rôle et la place de l'image dans la théorie jésuite des trois puissances de l'âme : mémoire, intelligence et volonté. Elle met en lumière, notamment, le rôle médiateur du phantasma dans la spiritualité qu'ils cultivent, notamment chez les auteurs qui s'inspirent d'Aristote et de saint Thomas, comme Louis de la Puente, Antoine Sucquet ou Jean Bourgeois. L'imagination intervient dans la composition de lieu ignatienne. La méditation se tient ainsi en équilibre entre le sensible et le spitituel (84). Il revient à Huguette Courtès, dans une très riche étude, d'éclairer, avec Descartes et Malebranche, la relation entre méditation philosophique et méditation spirituelle. Il est impossible de résumer une réflexion aussi dense. On retiendra surtout l'importance accordée par Descartes à l'attention, l'idée que la méditation cartésienne est marquée elle aussi par « des ruptures et des respirations » (111), et la nécessaire « lenteur » de sa progression (112). Quant à Malebranche, il enseigne qu'il faut rentrer en soi-même pour écouter le Christ-Verbe. Lui ausssi est un adepte de la lenteur. S'il croit à la raison, il demande surtout à la méditation de tracer le chemin du salut. En cela, il se rapproche des mystiques. Mais de son côté, Descartes, débarrassé de la caricature rationaliste que l'on faisait jadis de lui, pense en augustinien. « La contemplation $\mid$... ] occupe chez Descartes une place majeure, au point central et à la fin des Méditations : connaissance de soi, connaissance de Dieu, admiration du vrai bien, humilité retrouvée $\gg(132)$.

Terminons par la remarquable présentation de Christian Belin. C'est peu dire qu'il connaît bien son sujet. Avec lui, le XVII ème siècle apparaît dans toute sa complexité. La méditation est le reflet de celle-ci. Quoi de commun entre la spiritualité du Carmel et l'optimisme des Jésuites, entre Benoît de Canfeld et saint François de Sales ? Et que faire de Pascal, absolument inclassable ? Il reste que, chez les uns et chez les autres, la méditation est une « lecture tâtonnante et heuristique » (18) des textes de l'Ecriture ; qu'elle permet de « recueillir sa pensée » (21), de construire une « clôture intérieure » protégeant des corruptions du monde. Arrêtons-nous : 
le mieux est bien sûr de lire Christian Belin lui-même et toutes les communications qu'il a sollicitées. L'ensemble est vraiment d'une très haute tenue.

DANiEL MENAGER, Paris X-Nanterre

\section{Notes}

1. L'École du silence. Le Sentiment des images au XVIİ̀me siècle, Paris, Flammarion, 1994.

2. Peinture et dévotion en Europe du nord à la fin du Moyen Age, Paris : Flammarion, 1997.

\section{Francesco Petrarca}

\section{L'opera latina : tradizione e fortuna, actes $d u X V I^{e}$ colloque international} (Chianciano-Pienza, 19-22 juillet 2004)

Éd. Luisa Secchi Tarugi, « Quaderni della Rassegna », n 46

Florence, Franco Cesati Editore, 2006, 772 pages

Parmi les nombreux ouvrages liés aux manifestations commémorant le septième centenaire de la naissance de Pétrarque, à côté du recueil Petrarch and his Readers in the Renaissance (éd. K.A.E. Enenkel et J. Papy, « Intersections » ${ }^{\circ} 6$, Leyden : Brill, 2006), les actes du colloque consacré à l'œuvre latine de l'humaniste et du poète organisé à Chianciano et Pienza par Luisa Secchi Tarugi, et publiés par ses soins attentifs, méritent une attention toute particulière. Cet imposant volume en effet constitue à la fois une somme imposante sur la partie sinon la moins connue, du moins la plus diverse et la plus difficile d'une œuvre complexe, faite d'imitations et de références, mais aussi et plus généralement expression vivante d'une culture qui se révèle extraordinairement cohérente et qu'il serait oiseux de réduire à des composantes particulières. Pétrarque est d'abord un clerc, irréductible à la notion anachronique d'intellectuel (A. Romantchouk), dont l'activité lettrée se déploie dans tout l'espace de la vacatio et de l'otium : son œuvre, lieu d'affirmation du christianisme le plus exigeant, est aussi tout entière fondée sur une véritable passion pour une Antiquité qu'il cherche à comprendre, voire à restaurer, dans un effort permanent de synthèse mais aussi de justification ; il doit ainsi répondre à l'accusation de magie, liée à ses lectures classiques (B. Lavillatte). Les lettres, les Epistole sine nomine et les Familiari (dont G. Demerson et A. Piccardi étudient les emprunts et les citations), ne constituent pas seulement une œuvre parmi les autres, mais sont comme le lieu de condensation et de réflexion de la culture latine de Pétrarque dans son ensemble, témoignage des progrès conjoints d'une œuvre sans cesse reprise et de 